\title{
Propuesta didáctica para cambiar las ideas alternativas sobre la contribución de la fusión del hielo al aumento del nivel del mar
}

\author{
Jaime Carrascosa \\ Departamento de Didáctica de las Ciencias Experimentales. Universitat de València. \\ España. jaime.carrascosa@uv.es \\ ORCID: https://orcid.org/0000-0003-4707-1706 \\ Salvador Martínez \\ Instituto de Enseñanza Secundaria Cid Campeador. Valencia. España. \\ salvmart50@gmail.com \\ ORCID: https://orcid.org/0000-0002-0029-4825 \\ Manuel Alonso \\ Sección Local de Alicante de la Real Sociedad Española de Física. España. \\ manoloalonsosanchez@gmail.com \\ ORCID: https://orcid.org/0000-0002-6696-7623

\section{Juan José Ruiz Ruiz} \\ Departamento de Didáctica de las Ciencias Experimentales. Universitat de València. \\ España.Juan.J.Ruiz@uv.es \\ ORCID: https://orcid.org/0000-0002-0447-2393
}

[Recibido: 28 Marzo 2020. Revisado: 28 Julio 2020. Aceptado: 7 Septiembre 2020]

\begin{abstract}
Resumen: En la actualidad existen muchos estudios acerca del conocimiento teórico de la población sobre el cambio climático. Los resultados de estos estudios muestran la existencia de confusiones e ideas alternativas respecto a determinados fenómenos y conceptos básicos de gran importancia, las cuales constituyen un serio obstáculo para el aprendizaje en educación ambiental. En este trabajo se analiza una de esas ideas, relacionada con el impacto directo que tiene la fusión del hielo flotante en el aumento del nivel del mar. Concretamente, se muestra una primera aproximación respecto a su extensión y fortaleza entre alumnado de secundaria y profesorado en formación, detallando a su vez una estrategia didáctica, basada en la resolución de un problema como investigación, con la que conseguir su cambio o evolución hacia la idea científicamente correcta. En dicha estrategia se integran de manera funcional, contenidos teóricos, experimentación y nuevas tecnologías.
\end{abstract}

Palabras clave: Aumento nivel del mar; Educación ambiental; Fusión del hielo; Hielo continental y marino; Ideas alternativas.

Didactic proposal to change misconceptions about the contribution of the fusion of the ice to the rise in sea level

Abstract: Many studies on the theoretical knowledge that the population has about climate change are now available. The results of some of these studies show the existence of confusions and alternative ideas regarding certain phenomena and basic concepts of great importance, which constitute a serious obstacle to learning in environmental education. In this paper, one of these ideas, related to the direct impact that the melting of floating ice has on the increase of sea level is studied. Specifically, a first approach is shown regarding its extension and strength among secondary school students and teachers in training. An educational strategy to achieve its change towards the scientifically correct idea, based on the resolution of a problem is also detailed. In such strategy, theoretical contents, experimentation and new technologies are integrated in a functional way.

Keywords: Sea level rise; Environmental education; Ice fusion; Continental and sea ice; Misconceptions; Didactic proposal. 
Para citar este artículo: Carrascosa, J., Martínez, S., Alonso, M. y Ruiz, J. J. (2020) Propuesta didáctica para cambiar las ideas alternativas sobre la contribución de la fusión del hielo al aumento del nivel del mar.

Revista de Educación Ambiental y Sostenibilidad 2(2), $2301 . \quad$ doi: 10.25267/Rev_educ_ambient_sostenibilidad.2020.v2.i2.2301

\section{Introducción}

Tal y como se viene insistiendo en los sucesivos informes de evaluación que periódicamente elabora el grupo de científicos que conforman el Panel Intergubernamental sobre Cambio Climático (IPCC), la crisis climática es uno de los problemas más graves y urgentes a los que se enfrenta hoy la humanidad. Afortunadamente, se conocen las causas principales del problema y se dispone de los medios necesarios para hacerle frente; sin embargo, avanzar de forma realmente efectiva en su mitigación y en el desarrollo de medidas de adaptación, requiere, entre otras cosas, llevar a cabo grandes cambios que afectan fundamentalmente a la energía y recursos que utilizamos, así como al modelo económico imperante (Gil-Pérez y Vilches, 2019). Para que esos cambios puedan producirse, es necesario que exista una ciudadanía bien informada que los demande y un gran consenso social que favorezca su implantación. Es aquí donde la educación ambiental para el desarrollo sostenible juega un papel fundamental, tanto la que se realiza en la escuela, como la que proviene de los medios de comunicación o de las propias familias (Vilches y Gil-Pérez, 2016).

La educación ambiental ha de servir también para cuestionar y cambiar algunas concepciones alternativas relacionadas con problemas tan importantes como el Cambio Climático. Se trata de ideas asociadas a una particular interpretación de un concepto o fenómeno dado, muy diferente a la interpretación científica. Dichas ideas se caracterizan, fundamentalmente, por su amplia extensión (afectan a estudiantes de distintos niveles educativos y a una gran parte de la población en general) y por que quienes las detentan suelen estar bastante convencidos acerca de su validez (Arslan, Cigdemoglu y Moseley, 2012). A modo de ejemplo, podemos citar la confusión entre los conceptos de Tiempo (meteorología) y Clima (Heras, 2015), consistente en asociar ambos conceptos. Esta identificación errónea ha sido utilizada a veces, de forma consciente e interesada, para poner públicamente en duda la existencia del calentamiento global, aprovechando la ocurrencia, en una región y momento dados, de grandes nevadas o episodios de frío extremo (Bedford, 2010). Junto con esta idea alternativa, coexisten otras, tales como: pensar que el dióxido de carbono es el gas que más contribuye al efecto invernadero, identificar el adelgazamiento de la capa de ozono como una de las causas más importantes del Cambio Climático (Meira, 2015), o el convencimiento de que la fusión de todo el hielo flotante haría aumentar en muchos metros el nivel del mar (Schlichting, 2019). Este trabajo se centra, precisamente, en estudiar esta última idea $y$, sobre todo, en proponer una estrategia de enseñanza que facilite su cambio o evolución hacia la idea científicamente correcta. 


\section{Incidencia de las ideas alternativas sobre el impacto de la fusión del hielo en el nivel del mar}

En el caso de la influencia de la fusión del hielo en el nivel del mar, existen dos ideas alternativas que, curiosamente, se contraponen. La primera consiste en creer que la fusión de todo el hielo flotante en los océanos tendría como consecuencia directa un gran aumento del nivel del mar, produciendo extensas inundaciones. Hemos observado esta idea a lo largo de los últimos cursos en actividades para detectar ideas previas de nuestro alumnado de secundaria y de magisterio. Naturalmente esto no es correcto, ya que hablamos de hielo flotante y no de hielo continental. Quizá, el origen de esta idea haya que buscarlo en una generalización acrítica de las noticias que al respecto suelen darse en los distintos medios de comunicación, referidas a la pérdida de hielo continental en zonas como Groenlandia o la Antártida. Esto, junto con otras, referidas a la disminución de la extensión de hielo en el océano Ártico, puede conducir a establecer dicha generalización. La segunda idea alternativa lleva a pensar que si todo el hielo flotante se fundiese, este hecho, por sí mismo, no tendría ningún efecto directo en el nivel del mar (Noerdlinger y Brower, 2007; Villarroel-Villamor y Rey-Baltar, 2015). El origen de esta idea (también errónea), se debe a otra generalización, según la cual daría igual que el hielo flotara sobre agua dulce o que lo hiciera sobre agua salada, donde la densidad del agua proveniente de la fusión del hielo es menor que la del agua salada.

Nuestra hipótesis al comenzar este trabajo fue que la primera de las ideas alternativas expuestas se halla mucho más extendida entre el alumnado que la segunda. Con el fin de medir la incidencia de ambas ideas alternativas utilizamos las dos cuestiones siguientes:

A-Imagina que todo el hielo que hay flotando sobre las aguas del océano Ártico (Polo Norte) y del océano Antártico (Polo Sur), se fundiera transformándose en agua líquida. ¿Qué consecuencias directas importantes crees que tendría en el planeta la fusión de esa inmensa cantidad de hielo flotante?

B-Supongamos que todo el hielo que hay flotando en las aguas del océano Ártico (Polo Norte) y del océano Antártico (Polo Sur), se fundiese totalmente convirtiéndose en agua líquida. Si eso se produjese, el nivel del mar (subraya la opción con la que estés más de acuerdo):

a) No subiría nada

b) Subiría mucho menos de un metro

c) Subiría entre uno y diez metros

d) Subiría mucho más de diez metros

Como vemos, la cuestión A es totalmente abierta y en ella se hace hincapié en que se trata de hielo flotante, repitiendo este calificativo dos veces. En la cuestión B, el alumnado ha de escoger la opción que consideren más correcta de entre las cuatro que se ofrecen. Las dos cuestiones forman parte de dos cuestionarios distintos más amplios (la cuestión A correspondía a uno de los cuestionarios y la B al otro) que incluían más preguntas referidas a otras ideas alternativas relacionadas con el Cambio Climático y que fueron pasados durante los cursos académicos 18-19 y 19-20 entre estudiantes de Educación Secundaria Obligatoria (112 de 3ํㅡㄹ ESO y 137 de 4은 ESO) y de Magisterio (125 estudiantes de $2^{\circ}$ curso), todos ellos de centros públicos. El 
encuestador repartía aleatoriamente un cuestionario a la mitad de cada grupo encuestado y el otro al resto, y así cada estudiante de cada grupo solo cumplimentaba uno de los dos cuestionarios (de forma anónima y sin límite de tiempo). A todos los sujetos encuestados se les pedía también que calificasen cada una de sus respuestas con una nota entre 0 y 10 según la seguridad que tuviesen de que fuese científicamente correcta. Los resultados obtenidos para la cuestión A se recogen en la tabla siguiente (Tabla 1 ).

Tabla 1. Cuestión A. Porcentaje de respuestas erróneas y nota media de seguridad (sobre 10)

\begin{tabular}{|c|c|c|c|}
\hline Curso & $\begin{array}{l}\text { N Alumnos } \\
\text { encuestados }\end{array}$ & $\begin{array}{c}\text { ANM (\%) Aumento } \\
\text { del nivel del mar }\end{array}$ & X Nota media de seguridad \\
\hline $3^{\circ} \mathrm{ESO}$ & 56 & 89,3 & 8,8 \\
\hline $4^{\circ} \mathrm{ESO}$ & 69 & 86,9 & 8,4 \\
\hline $2^{\circ}$ Magisterio & 62 & 82,3 & 8,1 \\
\hline
\end{tabular}

En la tabla anterior, en la columna encabezada por ANM, se dan los porcentajes (\%) de alumnos (sobre el total, $\mathrm{N}$, a los que se les pasó la cuestión $\mathrm{A}$ ) de cada nivel educativo, que señalan, como primera consecuencia directa de la fusión de todo el hielo flotante, un aumento del nivel del mar (ANM). A la derecha de la misma se da la nota media de seguridad (x) correspondiente (sobre el total de alumnos que calificaron su respuesta) en cada caso.

En la tabla 2 se dan los porcentajes de alumnos que han escogido cada una de las opciones propuestas como respuesta correcta a la cuestión B y a su derecha la nota media de seguridad correspondiente (x). En el caso de la primera opción, "a", no aparece nota media de seguridad puesto que nadie se decantó por ella.

Los resultados obtenidos para ambas cuestiones son coherentes, en la cuestión A el porcentaje de estudiantes que considera que se produciría un gran aumento del nivel del mar supera para todos los niveles el 80\%, mientras que en la cuestión B el porcentaje de alumnado que considera que el aumento del nivel del mar será superior a 1 metro (suma de opción "c" y "d") supera el 90\%. siendo muchos más (en todos los niveles) quienes creen que el aumento sería mucho mayor de $10 \mathrm{~m}$. Además, las notas medias de seguridad con que se mantiene esta idea son muy altas (en general, entre 7 y 9).

Tabla 2. Cuestión B. Porcentaje de respuestas por opción y nota media de seguridad (sobre 10)

\begin{tabular}{|c|c|c|c|c|c|c|c|c|c|}
\hline Curso & $\mathrm{N}$ & $\begin{array}{l}0(\%) \\
\text { a) nada }\end{array}$ & $\mathrm{x}$ & $\begin{array}{c}<1(\%) \\
\text { b) menos de } 1 \mathrm{~m}\end{array}$ & $\mathrm{x}$ & $\begin{array}{c}1-10(\%) \\
\text { c) entre } 1 \text { y } 10 \mathrm{~m}\end{array}$ & $\mathrm{x}$ & $\begin{array}{c}>10(\%) \\
\text { d) mucho más de } 10 \\
\mathrm{~m}\end{array}$ & $\mathrm{x}$ \\
\hline 3을 ESO & 56 & 0 & - & 5,4 & 7,0 & 21,4 & 7,7 & 73,2 & 8,4 \\
\hline $4^{\circ} \mathrm{o}$ ESO & 68 & 0 & - & 3,4 & 6,0 & 42,9 & 7,3 & 53,7 & 7,5 \\
\hline $2^{\circ}$-Magisterio & 63 & 0 & - & 2,3 & 6,0 & 32,6 & 6,6 & 65,1 & 8,1 \\
\hline
\end{tabular}

Naturalmente, se trata tan solo de una primera aproximación y se precisa de más investigaciones con más estudiantes y en otros contextos, para determinar en qué medida se pueden generalizar los resultados aquí obtenidos. No obstante, estos son suficientes para justificar la necesidad de propuestas didácticas, como la que aquí se presenta, que permitan un tratamiento adecuado de este tipo de ideas. 


\section{Estrategia de enseñanza para clarificar la influencia de la fusión del hielo en el nivel del mar}

El estudio de las ideas alternativas del alumnado ha sido durante mucho tiempo una de las primeras líneas de investigación didáctica. No solo primera en el tiempo (contribuyendo al desarrollo de la Didáctica de las Ciencias Experimentales como un cuerpo específico de conocimientos), sino también primera en importancia, por la relevancia y cantidad de trabajos publicados en este campo (Carrascosa, 2005), donde destacan, con todo merecimiento, nombres como los de las investigadoras Rosalind Driver y Laurence Viennot. Los resultados de dichos trabajos muestran, reiteradamente, que el cambio conceptual que implica sustituir las ideas alternativas por las ideas científicas que se quieren enseñar, requiere de un cambio metodológico en el que se impulse el desarrollo de la competencia científica entre el alumnado (GilPérez y Carrascosa, 1994). Ello supone, entre otras cosas, plantear problemas de interés y saber precisarlos, emitir hipótesis y proponer posibles diseños para contrastarlas, llevar a cabo los diseños elaborados, realizar análisis críticos, interpretar, argumentar, modelizar, búsqueda de coherencia y globalidad, considerar nuevas perspectivas abiertas, etc. Como es lógico, estas actividades, para que puedan ser realmente efectivas, deberían potenciarse en todos aquellos aspectos que resultan clave para la enseñanza y el aprendizaje de las ciencias experimentales, como son los trabajos prácticos, la introducción de conceptos teóricos, la resolución de problemas y la propia evaluación. Concretamente, en este trabajo, se propone utilizar la resolución de un problema planteado como una pequeña investigación (MartínezTorregrosa, Gil-Pérez, Becerra-Labra y Guisasola, 2018), como estrategia adecuada para cambiar las ideas alternativas respecto a la influencia de la fusión del hielo flotante en el nivel del mar, anteriormente expuestas. Este problema puede utilizarse con estudiantes que ya conozcan el principio de Arquímedes y sus implicaciones en la flotabilidad de un objeto en el seno de un fluido. Se puede encontrar más información y más ejemplos de problemas de física y de química resueltos con la misma metodología, en la página web: https://didacticafisicaquimica.es/.

\section{Planteamiento del problema}

El problema se presenta al alumnado en dos etapas, la primera de las cuales consiste en pedir que se conteste la siguiente cuestión:

Supongamos un enorme bloque de hielo flotando en medio de un pequeño lago de agua dulce a comienzos del verano. Si toda esa masa de hielo flotante se fundiese, se puede afirmar que, debido a este hecho, el nivel del agua del lago (elige una de las opciones siguientes):

\section{a) Disminuiría}

b) Permanecería igual que antes

c) Aumentaría

La respuesta científicamente correcta a esta cuestión es la "b": el volumen total de agua líquida que se genera, en este caso, es justamente igual al volumen de la parte sumergida del bloque de hielo y, consecuentemente, el nivel del agua del lago no debería variar por esta causa ${ }^{1}$. Sin embargo, una gran mayoría de estudiantes suele

\footnotetext{
${ }^{1}$ Naturalmente, sí podría variar por otras causas como la evaporación, aportes externos, dilatación del agua por aumento de la temperatura, etc.
} 
escoger inicialmente la opción c. Por tanto, con esta pregunta previa, se pretende plantear una actividad de conflicto cognoscitivo, ya que una vez realizada, les pedimos que resuelvan cuantitativamente el problema y que utilicen el resultado obtenido para confirmar o cambiar su respuesta inicial a esta cuestión. El enunciado de dicho problema es:

Un bloque de hielo cuyo volumen total es de $100 \mathrm{~m}^{3}$ se halla flotando en medio de un lago de agua dulce. Se pide:

a) Volumen de hielo sumergido.

b) Volumen de agua líquida que produce el bloque de hielo cuando se funde totalmente.

Densidad agua dulce líquida: $d_{a}=1000 \mathrm{~kg} / \mathrm{m}^{3}$, densidad del hielo: $d_{h}=916^{\prime} 8 \mathrm{~kg} / \mathrm{m}^{3}$

Comenzamos el desarrollo del problema realizando un análisis cualitativo de la situación, en el que se precisan las condiciones que se consideran imperantes y qué es exactamente lo que se pide. Tal como se ilustra en la figura 1 adjunta, consideraremos un bloque de hielo parcialmente sumergido y usaremos la siguiente nomenclatura: Volumen total del bloque de hielo: $V_{T}$, volumen de la parte de hielo sumergida: $V_{s}$, densidad del agua dulce líquida: $d_{a}$, densidad del hielo: $d_{h}$.

De acuerdo con el principio de Arquímedes, el empuje experimentado por el hielo es una fuerza vertical y hacia arriba, de valor igual al peso del volumen de agua que desaloja (el volumen de agua desalojada $V_{\mathrm{a}}$ coincide exactamente, como es lógico, con el volumen de hielo sumergido $V_{s}$, es decir: $V_{a}=V_{s}$ ).

Por otra parte, al encontrarse el bloque de hielo en equilibrio, los módulos de la fuerza de empuje $\vec{E}$ y de la fuerza peso $\vec{P}$, han de ser iguales $(\mathrm{E}=\mathrm{P})$. En la figura 1 se han representado esquemáticamente ambas fuerzas.

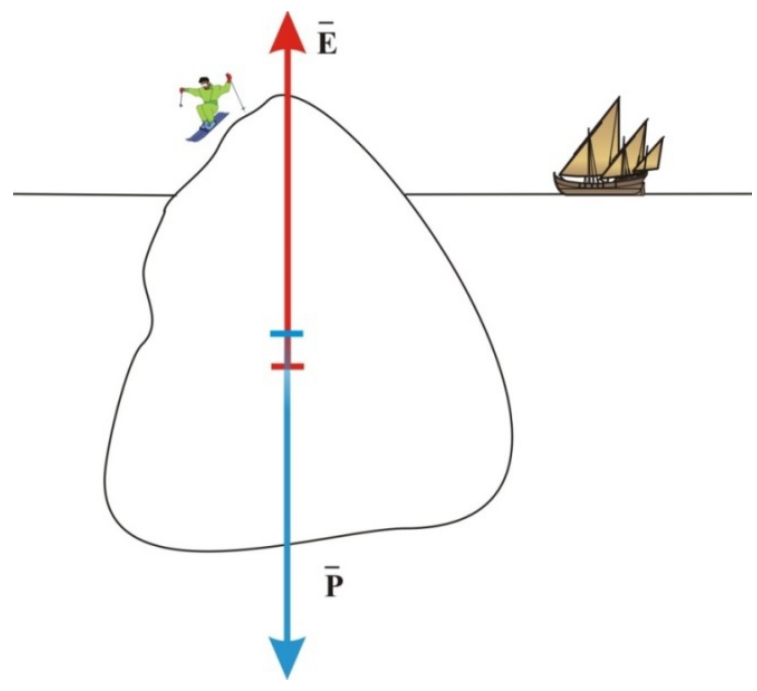

Figura 1. Fuerzas que actúan sobre un bloque de hielo flotante. (Fuente: elaboración propia).

Estamos, ahora, en condiciones de plantearnos cuánto valdrá el volumen de hielo sumergido $\mathrm{V}_{\mathrm{s}}$ 


\section{Hipótesis}

Una vez planteado el problema, pasamos a emitir hipótesis sobre los factores de los que puede depender la magnitud buscada. Esta es una actividad fundamental en el proceso de investigación, que orienta la resolución del problema y que también puede poner en evidencia, de manera funcional, posibles ideas alternativas, que deberán ser valoradas y contrastadas posteriormente a la luz del resultado que se obtenga.

En este caso, es lógico suponer que el volumen sumergido de hielo, $\mathrm{V}_{\mathrm{s}}$, dependa de la densidad del hielo, $d_{h}$, de la densidad del agua del lago, $d_{a}$, y del volumen total del bloque de hielo, $\mathrm{V}_{\mathrm{T}}$ :

$$
V_{s}=f\left(d_{h}, d_{a}, V_{T}\right)
$$

Concretamente, esperamos que $\mathrm{V}_{\mathrm{s}}$ aumente cuando (a igualdad de los restantes factores):

- La densidad del agua líquida del lago $\left(\mathrm{d}_{\mathrm{a}}\right)$ disminuya

La densidad del hielo $\left(\mathrm{d}_{\mathrm{h}}\right)$ aumente

El volumen total de todo el bloque de hielo $\left(\mathrm{V}_{\mathrm{T}}\right)$ aumente.

También podemos imaginar algún caso límite como, por ejemplo, que si $\mathrm{V}_{\mathrm{T}} \rightarrow 0$ el volumen de parte sumergida $V_{s}$ también tenderá a 0 , o bien que si la densidad del hielo pudiera, hipotéticamente, aumentar aproximándose a la densidad del agua líquida del lago $\left(\mathrm{d}_{\mathrm{h}} \rightarrow \mathrm{d}_{\mathrm{a}}\right)$ el bloque sobresaldría cada vez menos y $\mathrm{V}_{\mathrm{s}} \rightarrow \mathrm{V}_{\mathrm{T}}$.

\section{Estrategias de resolución y resolución propiamente dicha}

Las fuerzas que se ejercen sobre el bloque de hielo son el empuje y el peso. Ambas fuerzas dependen de los factores predichos en las hipótesis y, como el bloque de hielo flota en una posición de equilibrio estático, la resultante de ambas debe ser igual a cero. Por tanto, para resolver operativamente el problema bastará con igualar los módulos de ambas fuerzas y despejar la magnitud buscada, $\mathrm{V}_{\mathrm{s}}$ :

$$
\mathrm{E}=\mathrm{P} \rightarrow \mathrm{V}_{\mathrm{s}} \cdot \mathrm{d}_{\mathrm{a}} \cdot \mathrm{g}=\mathrm{V}_{\mathrm{T}} \cdot \mathrm{d}_{\mathrm{h}} \cdot \mathrm{g} \rightarrow V_{s}=\left(\frac{d_{h}}{d_{a}}\right) \cdot V_{T}
$$

Si analizamos el resultado anterior, vemos que no solo es dimensionalmente homogéneo (condición imprescindible, aunque no suficiente, para aceptarlo), sino que, además, en él se contemplan todas las hipótesis y casos límite considerados.

Sustituyendo valores:

$$
V_{s}=\left(\frac{916,8}{1000}\right) \cdot 100 \rightarrow \mathrm{V}_{\mathrm{s}}=91,68 \mathrm{~m}^{3}
$$

A partir de este resultado, para demostrar que el nivel del agua del lago no se altera si el hielo se funde completamente, planteamos:

¿Qué volumen $V_{a}$ de agua líquida produce el bloque de hielo cuando se funde totalmente?

De acuerdo con el principio de conservación de la masa, está claro que, aunque sus volúmenes sean distintos, la masa de agua líquida y la masa de hielo del cual procede han de ser iguales $\left(m_{a}=m_{h}\right)$, de modo que expresando cada una de estas masas en función de la densidad y volumen correspondiente, se puede obtener el volumen de agua líquida, $\mathrm{V}_{\mathrm{a}}$, pedido: 


$$
\mathrm{m}_{\mathrm{a}}=\mathrm{m}_{\mathrm{h}} \rightarrow \mathrm{d}_{\mathrm{a}} \cdot \mathrm{V}_{\mathrm{a}}=\mathrm{d}_{\mathrm{h}} \cdot \mathrm{V}_{\mathrm{T}} \rightarrow \quad V_{a}=\frac{d_{h} \cdot V_{T}}{d_{a}}
$$

Con las ecuaciones (1) y (2), comprobamos que $V_{a}=V_{s}$

Sustituyendo valores:

$$
V_{a}=\frac{916,8 \cdot 100}{1000}=91,68 \mathrm{~m}^{3}
$$

Así pues, el volumen de agua líquida que produce la fusión total del hielo flotante, cuando se trata de agua dulce, coincide exactamente con el volumen de la parte sumergida de dicho hielo. Vale la pena darse cuenta de que (1) y (2) son iguales porque la densidad del agua del lago (ecuación 1) y la densidad del agua resultante de la fusión del hielo (ecuación 2), también lo son (por eso hemos utilizado para ambas el mismo símbolo, $\mathrm{d}_{\mathrm{a}}$ ).

Por tanto, en estas condiciones, se puede afirmar que:

El hecho de que se funda el bloque de hielo flotante en un lago no tiene un efecto directo en el nivel del agua del lago.

A esta misma conclusión se puede llegar de forma cualitativa mediante una estrategia experimental, fácil de llevar a cabo en cualquier laboratorio escolar. Como inicio de la misma, se puede plantear al alumnado que propongan una posible experiencia destinada a mostrar que la fusión de hielo flotante sobre el agua dulce no altera el nivel de la misma. La realización de esta actividad por los alumnos distribuidos en pequeños grupos de trabajo, junto con la orientación del profesor, suele conducir a la propuesta de utilizar diversos recipientes como botellas o probetas grandes y trozos de hielo adecuados. Basta con, por ejemplo, introducir el trozo de hielo en una de tales probetas y añadir después agua líquida hasta un determinado nivel para comprobar que, una vez fundido totalmente el trozo de hielo, el nivel sigue siendo el mismo que inicialmente.

\section{Generalización del resultado. Fusión de bloques de hielo flotantes en el mar}

Una vez que hemos resuelto el problema para el caso de que el hielo flote en agua dulce, estamos en condiciones de dar un paso más y plantear la cuestión que realmente nos preocupa: ¿Ocurre lo mismo cuando hablamos de un gran bloque de hielo flotando en el océano?

Se trata de una pregunta muy importante, ya que, si no ocurriese lo mismo y resultase que el agua generada por la fusión del bloque de hielo ocupase más volumen que la parte sumergida del bloque de hielo que la originó, tendríamos que la fusión del hielo del casquete polar ártico y de la banquisa de hielo antártica, debida al calentamiento global, sí que estaría contribuyendo directamente, en alguna medida, al aumento del nivel del mar. Y lo cierto es que tenemos razones para pensar que no ocurre lo mismo. En efecto: el agua salada es más densa que el agua dulce, por lo que una misma masa de hielo flota más en el mar que en un lago de agua dulce. Acabamos de ver que, en el caso del lago, el volumen de agua líquida generado coincide exactamente con el volumen de la parte de hielo sumergida. En el caso del mar, el volumen de agua generado será el mismo que en el caso del lago (recordemos que la masa de hielo es la misma en ambos), pero como el volumen de hielo sumergido en el mar es menor que en el lago de agua dulce, el agua líquida generada tendrá un volumen algo mayor. La diferencia entre ambos volúmenes $\Delta \mathrm{V}=\mathrm{V}_{\mathrm{a}}-\mathrm{V}_{\mathrm{s}}$ es el volumen de agua que puede, en 
principio, afectar al nivel del mar contribuyendo a su elevación. No obstante, antes de ningún tratamiento operativo, siempre que se pueda, conviene realizar alguna estimación cualitativa previa. En este caso, por ejemplo, se puede avanzar que esa diferencia de volumen no debe ser muy grande, ya que la diferencia entre la densidad del agua de mar y la densidad del agua dulce tampoco lo es.

Si llamamos $d_{a m}$ a la densidad del agua marina y aceptamos un valor medio de 1027 $\mathrm{kg} / \mathrm{m}^{3}$, calculad el volumen sumergido para un bloque de hielo cuyo volumen total sea de $100 \mathrm{~m}^{3}$.

Teniendo en cuenta que el bloque de hielo se encuentra en equilibrio, la fuerza de empuje y el peso han de tener el mismo módulo (y sentidos contrarios), así que procediendo igual que anteriormente, tendremos que:

$$
\mathrm{E}=\mathrm{P} \rightarrow \mathrm{V}_{\mathrm{s}} \cdot \mathrm{d}_{\mathrm{am}} \cdot \mathrm{g}=\mathrm{V}_{\mathrm{T}} \cdot \mathrm{d}_{\mathrm{h}} \cdot \mathrm{g} \rightarrow \quad V_{s}=\left(\frac{d_{h}}{d_{\mathrm{am}}}\right) \cdot V_{T}
$$

Y sustituyendo valores:

$$
V_{s}=\left(\frac{916,8}{1027}\right) \cdot 100 \rightarrow \mathrm{V}_{\mathrm{s}}=89,27 \mathrm{~m}^{3}
$$

Como puede verse, el hecho de que el agua de mar sea algo más densa que el agua dulce, implica que el volumen de hielo sumergido es algo menor que anteriormente (porque flota más) y, por tanto, también es algo menor que el volumen de agua líquida originado cuando se funde todo el bloque. Basta comparar las expresiones (2) y (3) para, sin necesidad de ningún cálculo, darse cuenta de ello. Como se habrá podido apreciar, se ha utilizado para $d_{h}$ el mismo valor que en el caso anterior (916'8 $\mathrm{kg} / \mathrm{m}^{3}$ ), sin distinguir si el bloque de hielo corresponde a un glaciar que desemboca en el mar (formado a partir de nieve caída) o bien se ha formado por congelación de agua marina (es decir, a partir de agua salada). Esta aproximación puede hacerse, porque debido a determinados procesos físicos, la densidad del hielo marino, con el tiempo, llega a ser muy parecida a la del hielo continental y cuando este hielo se funde, la densidad del agua líquida resultante, se puede considerar, en el caso que nos ocupa, como aproximadamente igual a la del agua dulce (Vrbka y Jungwirth, 2007; Villarroel y Zuazogoitia, 2016; Schlichting, 2019). No ocurre lo mismo con la densidad del agua marina, que, como sabemos, varía de unos lugares a otros.

¿En cuánto excede ahora el volumen de agua líquida producida al volumen de hielo sumergido?

Como es lógico este exceso vendrá dado por $\Delta \mathrm{V}=\mathrm{V}_{\mathrm{a}}-\mathrm{V}_{\mathrm{s}}$

Sustituyendo $V_{a}$ y $V_{s}$ por sus expresiones correspondientes (2) y (3), tenemos que:

$$
\Delta V=V_{a}-V_{s}=\left(\frac{d_{h}}{d_{a}}\right) \cdot V_{T}-\left(\frac{d_{h}}{d_{a m}}\right) \cdot V_{T}
$$

Y simplificando, obtenemos finalmente:

$$
\Delta V=d_{h} \cdot V_{T} \cdot\left(\frac{1}{d_{a}}-\frac{1}{d_{a m}}\right)
$$

0 , lo que es equivalente: $\Delta V=m_{h} \cdot\left(\frac{1}{d_{a}}-\frac{1}{d_{a m}}\right)$ 
Si analizamos el resultado literal obtenido, podemos darnos cuenta de que $\Delta V$ será nulo cuando las densidades del agua resultante de la fusión del hielo y del líquido en el que se encuentra flotando dicho hielo sean iguales (como ocurre, de hecho, cuando el hielo se encuentra en un lago de agua dulce). Si se trata de un bloque de hielo flotando en el mar, como $\mathrm{d}_{\mathrm{am}}>\mathrm{d}_{\mathrm{a}}$ ocurrirá que $\Delta \mathrm{V}>0$ y ese $\Delta \mathrm{V}$ es el que incide en el nivel del agua aumentándolo.

Si sustituimos los valores numéricos pertinentes en la expresión (4), obtenemos:

$$
\Delta \mathrm{V}=2,41 \mathrm{~m}^{3}
$$

A este mismo resultado numérico, se puede llegar directamente sin más que tener en cuenta que el bloque de hielo marino de $100 \mathrm{~m}^{3}$ de volumen genera, al fundirse totalmente, un determinado volumen de agua líquida, independientemente de cómo se haya originado o de dónde se encuentre. Como ese volumen ya se calculó anteriormente y era de $91,68 \mathrm{~m}^{3}$, basta una simple resta para obtener el $\Delta \mathrm{V}$ buscado:

$$
\Delta \mathrm{V}_{\mathrm{a}}=91,68-89,27=2,41 \mathrm{~m}^{3}
$$

Esta pequeña diferencia, insistimos, sí que afecta al aumento del nivel del mar, aunque, claro está, muchísimo menos que si se tratase de agua líquida procedente de un bloque de hielo continental. En efecto, acabamos de ver que $100 \mathrm{~m}^{3}$ de hielo continental producen $91,68 \mathrm{~m}^{3}$ de agua líquida y que toda esa agua, al ser vertida en el mar, actúa directamente aumentando el nivel de este, mientras que, del mismo volumen de hielo flotante, al fundirse totalmente, solo $2,41 \mathrm{~m}^{3}$ del agua líquida generada, inciden en el nivel del mar (el resto, hasta $91,68 \mathrm{~m}^{3}$, ocupa el mismo volumen que ocupaba la parte sumergida del hielo).

En conclusión: en términos de aumento del nivel del mar, la fusión de cada $\mathrm{m}^{3} \mathrm{de}$ hielo flotante equivale a un aporte extra de 0,0241 $\mathrm{m}^{3}$ de agua.

\section{Resolución experimental. Fusión de hielo flotante en agua salina.}

Otra posible estrategia para resolver este problema es optar por la vía experimental. Ello, además, es una forma de someter a prueba el resultado obtenido teóricamente. Al igual que en el caso anterior, se puede recurrir a la utilización de botellas de plástico o probetas grandes y trozos de hielo adecuados. Sin embargo, aquí, en lugar de utilizar agua líquida dulce, se utiliza una disolución saturada de sal en agua. Obviamente, su densidad no es la misma que la del agua de mar, pero el experimento sirve igualmente para comprobar que al introducir un trozo de hielo en una probeta grande y después añadir el agua salada hasta alcanzar un nivel determinado, una vez completada la fusión del hielo, el nivel del líquido, al contrario que en la experiencia anterior, no es el mismo que inicialmente, sino que ha aumentado. El hecho de utilizar una disolución saturada de sal en agua hace que, al ser la densidad de dicha disolución sensiblemente mayor que la densidad del agua de mar, el aumento de volumen sea más perceptible. En Villarroel-Villamor y Rey-Baltar (2015) se describe una experiencia de este tipo utilizando botellas de plástico de distinto tamaño. Por supuesto, esta comprobación, se puede hacer, incluso, de forma cuantitativa (Schlichting, 2019) y constatar que los resultados numéricos (el obtenido teóricamente y el obtenido experimentalmente), dentro del margen de error correspondiente, coinciden. 
En nuestro caso, se utilizó una probeta de $100 \mathrm{ml}$ (calibrada en $\mathrm{ml}$ ), en la que se introdujo un bloque cilíndrico de hielo de 28,87 g de masa (previamente fabricado con agua destilada) e inmediatamente después se vertió parte de una disolución saturada de sal común en agua destilada (densidad 1,18 g/ml) hasta enrasar a $85 \mathrm{ml}$. Una vez fundido totalmente el hielo, se volvió a medir el volumen de agua salada resultante en el interior de la probeta, el cual resultó ser de $89 \mathrm{ml}$, lo que supone un aumento de volumen $\Delta \mathrm{V}=4 \mathrm{ml}$. En la figura 2 siguiente se puede observar la probeta utilizada en un momento dado durante la fusión del hielo en su interior.

Introduciendo los datos experimentales en la ecuación 4 anterior (donde se ha sustituido $\mathrm{d}_{\mathrm{am}}$ por $\mathrm{d}_{\mathrm{as}}$ representando la densidad del agua salada):

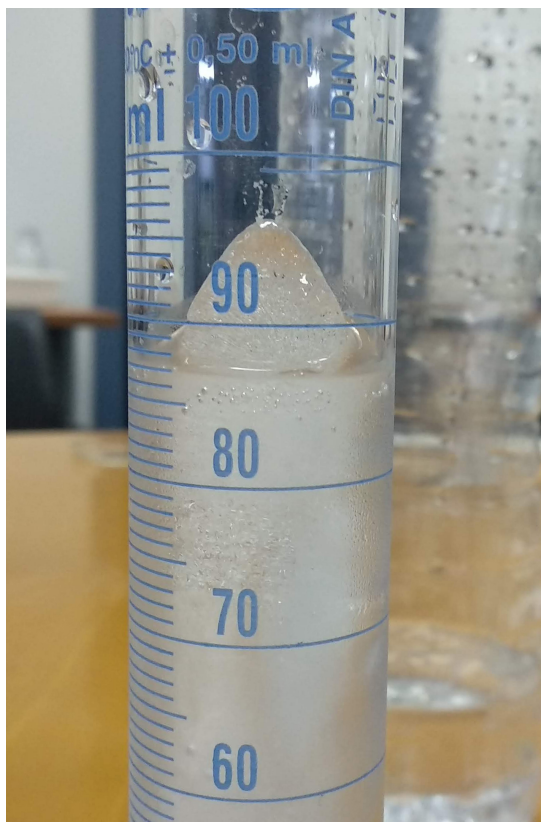

Figura 2. Bloque de hielo fundiéndose en una disolución de sal común en agua. (Fuente: elaboración propia)

Calculando la imprecisión absoluta correspondiente, se obtiene $\varepsilon(\Delta \mathrm{V})= \pm 0$ ' $5 \mathrm{ml}$, con lo que: $\Delta V=(4,4 \pm 0,5) \mathrm{ml}$. Como puede verse, el resultado experimental obtenido queda dentro del intervalo de imprecisión del resultado teórico.

\section{Perspectivas abiertas. Impacto global de la fusión del hielo continental y oceánico.}

Es posible preguntarse ahora por la intensidad de este efecto. Para ello, puede ser útil situarse en un escenario hipotético en el que se fundiese totalmente todo el hielo existente en el planeta, tanto continental como hielo marino flotante. Se pueden plantear las siguientes actividades:

Buscad los datos pertinentes y analizad cada caso (fusión del hielo continental y fusión del hielo marino) por separado, cuantificando y comparando la elevación del mar que se produciría.

No podemos extendernos aquí en la resolución concreta de esta actividad. No obstante, quienes estén interesados, pueden encontrarla totalmente resuelta en la página web: https://didacticafisicaquimica.es/, basta entrar en ella y seleccionar el tema: Del cambio climático hacia la construcción de un futuro sostenible, consultando 
el anexo 1 incluido al final de dicho tema. Aquí nos limitaremos a analizar los resultados que se obtienen:

En cuanto al aumento del nivel del mar que se produciría por la fusión de todo el hielo continental, este resultaría ser de entre $60 \mathrm{~m}$ y $70 \mathrm{~m}$ por encima del nivel actual y haría que el agua del mar se adentrase kilómetros en todos los continentes, cambiando totalmente la configuración de los mapas. Naturalmente, hemos considerado un proceso hipotético, pero conviene no olvidar que el hielo continental, a consecuencia del calentamiento global, se está fundiendo y que eso ya está contribuyendo de forma importante al aumento del nivel del mar, que ha sido de unos $16 \mathrm{~cm}$ en los últimos 100 años (IPCC, 2019), y a la disminución de su salinidad, ambas cosas de muy graves consecuencias para toda la humanidad y la biodiversidad en general.

El resultado es muy diferente cuando se considera la contribución directa al aumento del nivel del mar de la hipotética fusión de todo el hielo flotante. En este caso, dicho aumento estaría en torno a los $4 \mathrm{~cm}$. Así pues, la subida del nivel del mar, ocasionada directamente por la fusión de todo el hielo flotante (cualquiera que sea su origen) existente en un momento dado, se puede considerar despreciable si la comparamos con la subida ocasionada por la fusión del hielo continental asentado en tierra, por lo que, en lo que se refiere al calentamiento global, es perfectamente lícito ignorar la contribución directa de la fusión del hielo flotante al aumento del nivel del mar. No podemos ignorar, en cambio, su contribución indirecta, ya que, por ejemplo, la disminución progresiva de la superficie de ese enorme escudo protector que constituye el hielo flotante del Ártico, el cual refleja al espacio una gran parte de las radiaciones solares que llegan a la zona, además de otras consecuencias, hace que el agua se caliente más $\mathrm{y}$, por tanto, se expanda, y esa dilatación térmica sí que incide notablemente en el aumento del nivel del mar.

\section{Refuerzo. Animaciones interactivas para modelizar la fusión del hielo flotante.}

Para reforzar varios de los conceptos involucrados en este problema se han creado dos animaciones interactivas utilizando el programa Modellus (Alonso, 2007; Neves y Teodoro, 2010).

En una de ellas (figura 3), se representa en la pantalla un bloque de hielo flotando sobre una masa de agua. El alumnado puede usar un controlador manual para elegir entre agua marina y agua dulce, y otro para fijar el volumen total inicial del bloque de hielo flotante. La animación calcula y representa el porcentaje de hielo flotante, junto con el aumento del volumen de agua líquida que se produciría si se fundiera totalmente dicho bloque. Finalmente, la animación también permite obtener el incremento en el volumen y en el nivel del mar que tendría lugar si fundiera un porcentaje (que se puede determinar usando un tercer controlador manual) de todo el hielo flotante actual. La figura 3 muestra los resultados cuando se adoptan los datos que se han usado en el problema.

La otra animación (figura 4), remite a la estrategia de resolución del problema por vía experimental anteriormente descrita. Representa un trozo de hielo flotante en una probeta llena de una disolución salina, y se pueden usar tres controladores manuales para modificar la densidad de la disolución, la masa del pedazo de hielo y la propia densidad del hielo. Además de calcular los porcentajes y los volúmenes de hielo 
flotante y sumergido, la animación también obtiene el incremento de volumen que se produciría en la probeta si se derritiera el pedazo de hielo.

El alumnado puede realizar con esta segunda animación varias actividades interesantes e instructivas. Por ejemplo: puede comprobar que, al modificar la masa del pedazo de hielo flotante, este permanece con el mismo grado de hundimiento (en este caso no cambian los porcentajes de hielo sumergido y flotante, aunque sí obviamente, el volumen de ambos).
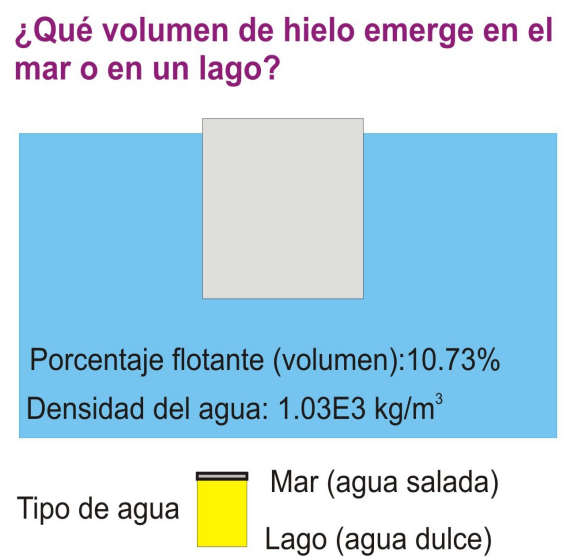

¿En cuánto aumenta el volumen y el nivel de agua si se funde hielo flotante?

Al fundirse en el mar un bloque de hielo:

Volumen del hielo $100 \mathrm{~m}^{3}$

Aumento de volumen del mar: $2.41 \mathrm{~m}^{3}$

Al fundirse en el mar un porcentaje dado del hielo flotante:

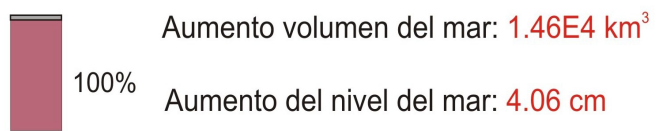

Figura 3. Bloque de hielo flotante en una masa de agua. (Fuente: elaboración propia).

También puede probar casos límite como, por ejemplo, el que se obtiene al igualar las densidades del agua y del hielo (el bloque se sumerge completamente); y, por supuesto, también puede comparar el resultado teórico del ascenso del nivel de líquido que se ha de producir si se derrite todo el hielo, con el obtenido en el laboratorio. La figura 4 muestra los resultados cuando se adoptan los datos obtenidos en la experiencia descrita anteriormente en el apartado 3.5.

¿Qué volumen del trozo de hielo está sumergido?

¿Cuanto aumenta el volumen si se derrite todo el hielo?

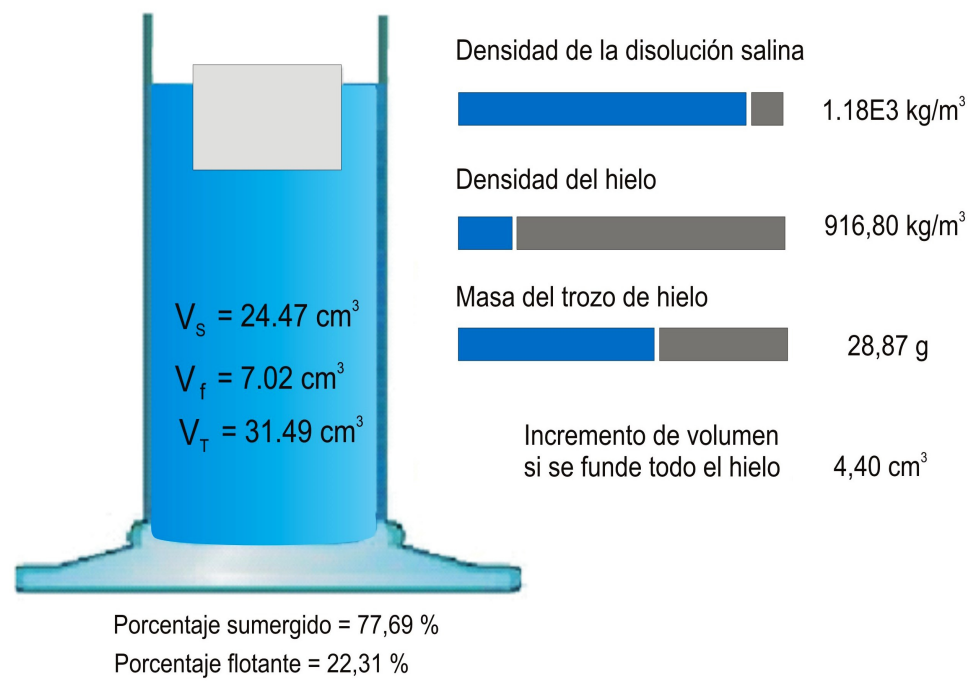

Figura 4. Trozo de hielo flotante en disolución acuosa de $\mathrm{NaCl}$. (Fuente: elaboración propia) 
Las dos animaciones y el programa para hacerlas correr están disponibles en la página Web de Materiales para la Enseñanza y la Divulgación de la Física, de la Sección Local de Alicante de la RSEF. http://rsefalicante.umh.es/fisica.htm

\section{Consideraciones finales}

En la comunidad científica existe un gran consenso respecto de la gravedad y la urgencia para afrontar el problema de la crisis climática actual. Se trata de un problema que preocupa a gran parte de la población (Meira, Arto, Heras, Iglesias, Lorenzo y Montero, 2013), los medios de comunicación le dedican cada vez mayor atención y las autoridades educativas lo han incluido en la mayoría de los currículos escolares. Cabe preguntarse si toda esta atención se ha traducido en una formación adecuada del alumnado y en qué medida se siguen detectando ideas alternativas capaces de sobrevivir a la formación. Temática importante, puesto que algunas de estas ideas son utilizadas por negacionistas para cuestionar el calentamiento global antropogénico (Redford, 2010; Sezen-Barrie, Shea y Borman, 2019).

En este trabajo, se intenta hacer una primera aproximación al estado de esta cuestión, analizando la extensión y la fortaleza de una idea alternativa ya conocida, según la cual la fusión de las banquisas e icebergs flotantes en el mar haría aumentar en muchos metros el nivel de este. Los primeros resultados obtenidos muestran que esta idea, a pesar de la educación ambiental que se imparte y de toda la información no académica en la que estamos inmersos, sigue estando muy presente y con un elevado grado de convencimiento tanto entre el alumnado de secundaria como en los futuros maestros que hemos encuestado. Por otra parte, el alumnado no utiliza los conocimientos estudiados en su formación reglada, puesto que ni utiliza argumentos basados en el conocimiento científico como el principio de Arquímedes (cuestión A), ni considera aumentos del nivel del mar mínimo o despreciable para la fusión total del hielo flotante, más coherente con las ideas científicas del currículo(cuestión B). Este hecho pone de manifiesto que el aprendizaje del alumnado en el tema de la flotabilidad no ha sido significativo. Además, esta situación se reproduce en todos los niveles estudiados $3^{\circ}$ y $4^{\circ}$ de ESO y $2^{\circ}$ de Magisterio. Naturalmente, con la pequeña muestra utilizada, no se pueden generalizar los resultados. No obstante, son lo suficientemente relevantes como para tratar de mejorarlos y esto es lo que se ha procurado hacer en el resto del trabajo: presentar una estrategia fundamentada con la que conseguir un aprendizaje realmente significativo. Esencialmente consiste en utilizar un problema planteado como una pequeña investigación, con el propósito explícito de impulsar y desarrollar la competencia científica. Por otra parte, en el problema aquí propuesto, se tratan conjuntamente, aspectos teóricos, prácticos y TIC. Dicho tratamiento conjunto, constituye un nuevo enfoque (más coherente con la actividad científica), que posibilita su implementación en diferentes niveles de enseñanza con mayor o menor grado de dificultad. Por ejemplo, en edades más tempranas se deberán omitir los desarrollos formales, pero será posible plantear el problema y resolverlo (en términos cualitativos) de forma experimental.

El trabajo puede continuarse, ampliando el número de encuestados (para ver en qué medida confirman o no estos primeros resultados) y desarrollando esta estrategia de enseñanza, en la medida de lo posible, también en la educación ambiental, prestando una atención particular a las confusiones e ideas alternativas más importantes que se detectan entre el alumnado. 


\section{Referencias bibliográficas}

Alonso, M. (2007). Animaciones Modellus y videos de experiencias de laboratorio para dar un nuevo impulso a la enseñanza de la mecánica newtoniana. Revista Electrónica de Enseñanza de las Ciencias, 6(3), 729-745.

Arslan, H. O. , Cigdemoglu, C., y Moseley, C. (2012). A Three-Tier Diagnostic Test to Assess Pre-Service Teachers' Misconceptions about Global Warming, Greenhouse Effect, Ozone Layer Depletion, and Acid Rain. International Journal of Science Education, 34(11), 1667-1686, DOI: 10.1080/09500693.2012.680618.

Bedford, D. (2010). Agnotology as a Teaching Tool: Learning Climate Science by Studying Misinformation. Journal of Geography, 109(4), 159-165 DOI: 10.1080/00221341.2010.498121.

Carrascosa, J. (2005). El problema de las concepciones alternativas en la actualidad: Análisis sobre las causas que las originan y/o mantienen Análisis sobre las causas que las originan y/o mantienen (Parte I). Revista Eureka sobre Enseñanza y Divulgación de las Ciencias, 2(2), 183-208. Recuperado a partir de https://revistas.uca.es/index.php/eureka/article/view/3918.

Gil-Pérez, D., y Vilches, A. (2019). La comprensión e impulso de la Sostenibilidad: un requisito imprescindible para una acción educativa y ciudadana eficaz. Revista de Educación Ambiental y Sostenibilidad 1(2), 2101. DOI: 10.25267/Rev_educ_ambient_sostenibilidad.2019.v1.i2.2101.

Gil-Pérez, D., y Carrascosa, J. (1994). Bringing Pupils' Learning Closer to a Scientific Construction of Knowledge: A Permanent Feature in Innovations in Science Teaching. Science Education, 78(3), 301-315.

Heras, F. (2015). La educación en tiempos de cambio climático. Mètode Science Studies Journal, 85, 57-63. DOI: 10.7203/metode.85.4220.

Martínez-Torregrosa, J., Gil-Pérez, D., Becerra-Labra, C., y Guisasola, J. (2018). ¿Podemos mejorar la enseñanza de la resolución de problemas de "lápiz y papel” en las aulas de Física y Química? Educación Química, 16(2), 230-245.

Meira, P. A., Arto, M., Heras, F., Iglesias, L., Lorenzo, J. J., y Montero, P. (2013). La respuesta de la sociedad española ante el cambio climático. 2013. Madrid: Fundación Mapfre.

Meira, P. A. (2015). ¿Hay un agujero en la capa de ozono de tu cambio climático? De la cultura científica a la cultura común. Mètode Science Studies Journal, 85, 49-55. DOI:10.7203/metode.85.4219.

Neves, R., y Teodoro, V. (2020). Enhancing Science and Mathematics Education with Computational Modelling. Journal of Mathematical Modelling and Application, 1(2), 2-15.

Noerdlinger, P. D., y Brower, K. R. (2007). The melting of floating ice raises the ocean level. Geophysical Journal International, 170, 145-150. DOI:10.1111/j.1365246X.2007.03472.x.

Panel Intergubernamental sobre Cambio Climático, IPCC, (2019). Resumen para 
responsables de políticas. En H. O. Pörtner, D. C. Roberts, V. Masson-Delmotte, P. Zhai, M. Tignor, E. Poloczanska, K. Mintenbeck, M. Nicolai, A. Okem, J. Petzold, B. Rama, y N. M. Weyer (eds.), Informe especial sobre los océanos y la criosfera en un clima cambiante del IPCC. En prensa. https://www.ipcc.ch/site/assets/uploads/sites/3/2020/07/SROCC_SPM_es.p d.

Schlichting, H. J. (2019). ¿Qué ocurriría si se fundieran todos los icebergs? Investigación y Ciencia, 519, 82-83.

Sezen-Barrie, A., Shea, N., y Borman, J. H. (2019). Probing into the sources of ignorance: science teachers' practices of constructing arguments or rebuttals to denialism of climate change, Environmental Education Research, 25(6), 846866, DOI: 10.1080/13504622.2017.1330949.

Vilches, A., y Gil-Pérez, D. (2016) La transición a la Sostenibilidad como objetivo urgente para la superación de la crisis sistémica actual. Revista Eureka sobre Enseñanza y Divulgación de las Ciencias, 13(2), 395-407. Recuperado de: http://hdl.handle.net/10498/18296.

Villarroel-Villamor, J. D., y Rey-Baltar, D. Z. (2015). El impacto de la fusión de los icebergs en el nivel del mar. Revista Eureka sobre Enseñanza y Divulgación de las Ciencias, 12(1), 178-185. Recuperado a partir de https://revistas.uca.es/index.php/eureka/article/view/2909.

Villarroel, J. D., y Zuazagoitia, D. (2016). The phenomenon of brine rejection: a practical proposal for prospective primary teacher training. Educación Química, 27(3), 205-208. https://doi.org/10.1016/j.eq.2016.03.001.

Vrbka, L., y Jungwirth, P. (2007). Molecular dynamics simulations of freezing of water and salt solutions. Journal of Molecular Liquids, 134(1), 64-70. 